\title{
Planning strategies for inter-fractional robustness in pancreatic patients treated with scanned carbon therapy
}

\author{
Vania Batista ${ }^{1 *}$ D, Daniel Richter ${ }^{2,5}$, Stephanie E. Combs ${ }^{1,4}$ and Oliver Jäkel $\left.\right|^{3,6}$
}

\begin{abstract}
Background: Managing inter-fractional anatomy changes is a challenging task in radiotherapy of pancreatic tumors, especially in scanned carbon-ion delivery. This treatment planning study aims to focus on clinically feasible solutions, such as the beam angle selection and margin design to increase the robustness against inter-fractional uncertainties.

Methods: This study included 10 patients with weekly 3D-CT imaging and physician-approved Clinical Target Volume (CTV). The study was directed to keep the CTV-coverage using six beam angle configurations in combination with different Internal Target Volume (ITV concepts. These were: geometric-margin (symmetric 3 and $5 \mathrm{~mm}$ margin); range-equivalent margins with an isotropic $\mathrm{HU}$ replacement; and to evaluate the need of asymmetric margins the water-equivalent range path (WEPL) was determined per patient from the set of CTs.

Plan optimization and forward dose calculation in each week-CT were performed with the research treatment planning system TRiP98 and the plan quality evaluated in terms of CTV coverage (V95 CTV) and homogeneity dose $\left(\mathrm{H}_{\mathrm{CTV}}=\mathrm{D} 5-\mathrm{D} 95\right)$.
\end{abstract}

Results: The beam geometry had a substantial impact on the target irradiation over the treatment course, with the single posterior or two beams showing the best average coverage of the CTV. The use of geometric margins for the more robust beam geometries showed acceptable results, with a V95 CTV of $(99.2 \pm 1.2) \%$ for the $5 \mathrm{~mm}$-margin. For the non-robust configurations, due to substantial changes in the radiological depth, the use of this margin results in a V95crv that might be below $80 \%$, only showing improvement when the range changes are included.

Conclusions: Selection of adequate beam configurations and treatment margins in ion-beam therapy of pancreatic tumors is of great importance. For a single posterior beam or two beam configurations, application of geometrical margins compensate for dose degradation induced by inter-fractional anatomy changes for the majority of the analyzed treatment fractions.

\section{Introduction}

Pancreatic cancer is still a disease without an effective treatment option usually with low survival rates and local control [1]. For locally advanced tumors one of the treatment schemes is photon radio-chemotherapy. However, a recent analysis showed that most tumor recurrences occur within a $2 \mathrm{~cm}$ radius of the primary tumor [2], indicating the need of dose escalation to improve

\footnotetext{
* Correspondence: vania.batista@med.uni-heidelberg.de

${ }^{1}$ Hospital for Radiooncology and Radiation Therapy, Heidelberg University Hospital, Heidelberg, Germany

Full list of author information is available at the end of the article
}

the local control while keeping reduced side-effects. Hence, an alternative is the use of ion-beam therapy with proton or heavy ions [3]. Studies from the National Institute for Radiological Sciences (NIRS) and at the Heidelberg Ion-Beam Therapy Center (HIT) investigate this assumption. NIRS studies about the dose escalation and combined-chemotherapy with carbon-ions radiotherapy have shown a strong increase in the tumor local control and in the overall survival [4]. These results have motivated the conduct of the Phoenix-Trial at HIT which intends to use scanned carbon-radiotherapy to downsize the tumor before surgery [5]. 
The physical characteristics of the carbon-ions beam offer the possibility for highly conformal treatments as consequence from the reduced lateral scattering, the finite range and the shape of the depth dose profile. Moreover, from a radiobiological point of view, carbonions exhibit a relative biological effectiveness (RBE) of 1.16-2.46 [6] for pancreatic tumor cells and a low oxygen enhancement ratio, which due to the large fraction of hypoxic cells in pancreatic tumors make them a promising treatment option [7].

However the finite range of ions is also a source of uncertainties. The high sensitivity of the ion range to density changes in the beam-path induces dose underand overshoots. These variations can either result from inter-fractional anatomy changes, patient positioning or intra-fractional motion [3].

Inter-fractional changes in pancreatic patients are mainly due to tumor shrinkage organ filling (bowel and stomach) and loss of adipose tissue [8]. These variations can be included in the planning process through the use of safety margins, although this compromises the dose to the normal tissues and does not consider range changes. The selection of the beam angles also affects plan quality and should avoid density variations along the beam path, minimizing the variations of the water-equivalent-path-length (WEPL). Note, that the effect of tumour shrinkage is not avoided by these methods, but its influence in the neighboring OARs can be reduced through the use of robust beam configurations, and its monitoring will help in the identification of the need for plan adaptation.

Intra-fractional motion is caused by respiration [9] and bowel movement [10] which besides range changes might results in under- and over-dosage regions, as result from the interference between the beam delivery and the target motion (interplay) in scanned beam delivery systems.

This study focuses on the impact of inter-fractional motion on the delivered dose during the pancreatic treatment and on the development of clinically feasible strategies to increase the treatment robustness by optimizing beam angles and internal margins. The investigation of the effects of intra-fraction motion it is not within the scope of this work and will be the subject of future publications.

\section{Methods}

\section{Patient dataset \& imaging}

For a set of ten patients which had already been treated with photon radiotherapy, an in-silico analysis of the impact of inter-fractional motion on the plan quality for the treatment with scanned carbon-ions was performed. All the patients were weekly CT imaged for positioning verification purposes.

The four CT images were registered to the first week-CT (considered here as the $\mathrm{CT}_{\text {plan }}$ ) through rigid registration.
Registration was validated using anatomical landmarks and visual inspection.

\section{Contouring and volume definition}

All weekly-CTs included physician-approved contours of the Gross Tumor Volume (GTV) and Clinical Target Volume (CTV). The CTV was defined as the GTV plus an isotropic margin of $5 \mathrm{~mm}$. Details on the initial CTV volume of each patient and its variation in volume and location over the treatment are presented in the Additional file 1: Table S.1. To incorporate inter-fractional motion effects we investigated three approaches to define an Internal Target Volume (ITV) for dose optimization:

- Geometrical ITV $\mathbf{G}_{\mathbf{G}}$ : Geometric concept by application of 3 and $5 \mathrm{~mm}$ symmetric margins to the CTV volumes, ITVG 3 and ITVG $_{5}$, respectively. Results were compared with the use of no additional margin $\left(\mathrm{ITV}_{\mathrm{G} 0}\right)$.

- Uniform range-margin $\mathbf{I T V}_{\mathbf{H U}}$ : Based on the geometric concept $\left(\mathrm{ITV}_{\mathrm{G}}\right)$ a uniform range-margin was introduced into the optimization by replacing the HU values in an isotropic CTV-ITV margin of 3 or $5 \mathrm{~mm}$ with the patient-specific median density of the pancreas. The volumes were defined as ITV $\mathrm{HU}_{3}$ and $\mathrm{ITV}_{\mathrm{HU}}$. In addition, also the areas inside the ITV $_{\mathrm{HU}}$, in which the density was less than twice the standard deviation of the median $\mathrm{HU}$ value, were overwritten.

- Beam specific Water-equivalent-path length, ITV $V_{\text {WEPL: }}$ To assess the needed margin to overcome the range uncertainties caused by anatomy variations along the treatment, an ITV considering the changes of the WEPL over the set of weekly-CTs, ITV WEPL, was determined. To this end, we employed the method defined by Graeff et al. [11], using the 4D extension of the treatment planning system (TPS) TRiP98 [12, 13], TRiP4D. In short, the HU changes along the specific beam direction in the set of weeklyCTs was converted into range changes, which led to


basis of these changes, to ensure adequate CTV coverage for the whole imaging set.

The definition of a Planning Target Volume (PTV) was out of the scope of this work as safety margins for set-up uncertainties will be subject of a separate investigation. Likewise intra-fractional motion uncertainties were also not considered.

\section{Treatment plan optimization and forward dose calculations}

Plans were optimized using the research TPS TRiP98 [13] where the same beam base data and RBE input tables [12] 
were used as for our commercial system in clinical use, Syngo ${ }^{\circ}$ RT Planning (Siemens Oncology Care Systems, Germany). The optimization technique used was intensity-modulated particle therapy (IMPT) with the ITV as the target to get full coverage of the CTV with a biological dose of $15 \times 3$ Gy (RBE). The biological dose calculation uses the local effect model [14]. The spinal cord, kidneys, stomach and bowel were considered organs-at-risk (OARs) and treatment plans were tailored to respect the tolerances suggested by the literature [15]. The $\alpha / \beta$-ratio equal to 2 was used for all the various organs.

The IMPT optimization was performed with a reduced number of constraints in a way to preserve relatively high dose homogeneity in the single beams, preventing high gradients in the target volume, and avoiding the need of patch techniques. In the Additional file $1 \mathrm{a}$ comparison between SFUD and IMPT plans for a set of patients is provided. This allows to demonstrate that IMPT plans with comparable homogeneity to SFUD plans and without compromising the plan quality can be achievable.

For each patient six different beam geometries were optimized (Fig. 1) using pencil beams with $10 \mathrm{~mm}$ of FWHM, a raster spacing of $3 \times 3 \mathrm{~mm}$ in lateral direction and an iso-energy slice spacing of $3 \mathrm{~mm}$. Our raster scanning technique is characterized by a synchrotron spill structure of $5 \mathrm{~s}$, a sweep time of $60 \mathrm{~m} / \mathrm{s}$ and no rescanning is currently implemented.

Based on the optimized plan for the $\mathrm{CT}_{\text {plan }}$ the forward calculation was performed for the following weeks by application of the raster-scan sequence on the registered CT.

\section{Data analysis}

The CTV coverage was determined by the volume that receives more than $95 \%$ of the prescribed dose $\left(\mathrm{V} 95_{\mathrm{CTV}}\right)$. Additionally the CTV homogeneity dose $\left(\mathrm{H}_{\mathrm{CTV}}\right)$ was calculated by the difference of the dose given to $5 \%$ and $95 \%$ of its volume, $\mathrm{H}_{\mathrm{CTV}}=\mathrm{D} 5$ - D95.
The concept of the ITV $V_{\text {WEPL }}$ was applied to evaluate the need of asymmetrically defined ITV to increase the plan robustness for patients under larger range changes. Using the TPS TRiP4D the ITV $\mathrm{WWPL}_{\mathrm{W}}$ was determined per field from the set of weekly-CTs and contours. The field-specific ITV $V_{\text {WEPL }}$ was evaluated by comparison with the CTV and $\mathrm{ITV}_{\mathrm{G} 5}$, through the volumetric changes and the ratio of the overlapping volumes (Dice Similarity Coefficient, DSC [16]). To extract the information of the margins direction and size we evaluated the variation of the center of mass $(\mathrm{COM})$ and the Hausdorff distance (HD) between ITV WEPL $_{\text {and CTV [16], which }}$ identifies the largest of all distances from one of the contours to the closest point on the other contour [17]. To reduce the patient-specific influence when applying this concept to other patients, the $95^{\text {th }}$ percentile of the $\mathrm{HD}$ was used as evaluation metric, $\mathrm{HD}_{95}$.

\section{Results}

The impact of anatomy and range changes on the target coverage and dose homogeneity among beam geometries margins concepts, patients, and fractions were analyzed. Furthermore, the CTV and COM changes along the treatment were analyzed and used to identify the reason for the dose degradation (e.g. CTV shrinkage, shift of CTV $\mathrm{COM}$ due to bowel volume, etc.). These data are available in the Additional file 1. Figure 2 shows two examples of the observed anatomical changes.

The plans with multiple beams showed an equally distributed and homogenous dose per beam i.e. each beam contributes to the total dose with $(50.0 \pm 2.5) \%$ of the number of particles and a homogenous dose distribution per beam is observed, as consequence of a moderate IMPT optimization.

\section{Inter-fractional changes and Beam Geometry Robustness} The beam geometry showed a substantial impact on target coverage and homogeneity over the treatment course with the configuration C and D (Figs. 1 and 3) showing

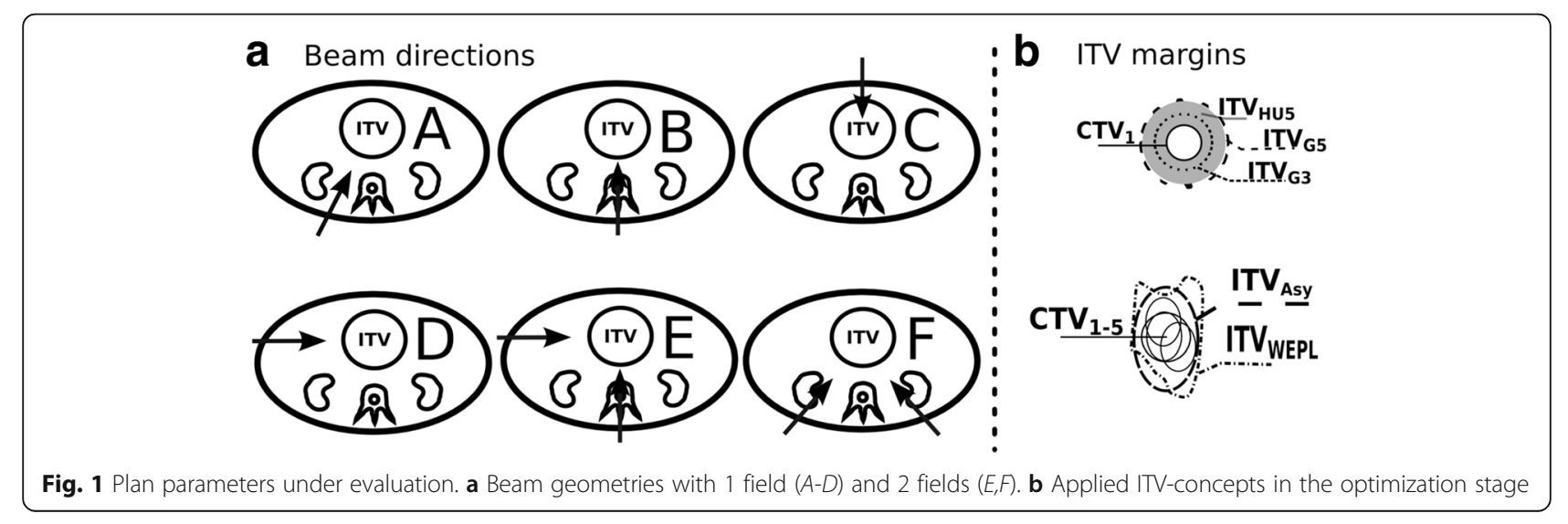




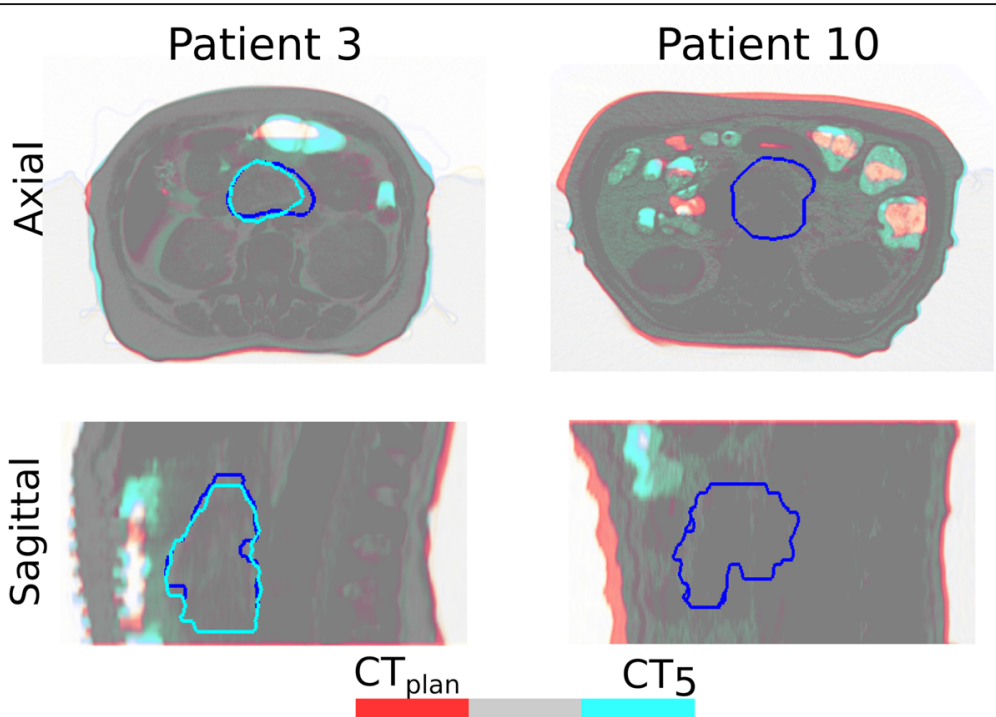

Fig. 2 Overlay of the planning $C T$ (red) and the CT of the week 5 (blue) for the patient 3 and 10. The grey color means perfect overlay between the patient anatomy and the red/blue color the region with anatomy changes (i.e. bowel, stomach and weight loss). The dark blue contour represents the planning CTV, while the light blue the CTV of the week 5 CT

the worst average coverage as well as standard deviations along the treatment (Table 1). These values are related with the observed range uncertainties in the anterior abdomen region due to the inter-fractional variability of the anatomy (bowel, stomach, weight loss). Figure 3 shows the dose distribution impact in one of the follow-up CTs for four patients as representative of these inter-fractional changes contributors. V95 ${ }_{\mathrm{CTV}}$ for non-robust geometries decreased by up to $30 \%$ and $28 \%$, for a $3 \mathrm{~mm}$ and $5 \mathrm{~mm}$ margin, respectively. This extreme effect was detected, as example, in a patient with larger weight loss (patient 8 , Fig. 3d) and in a case with strong bowel variation (e.g. patient 3 and 10, Fig. 3a and c).

\section{Impact of margins}

The selection of robust beam geometries (as A B, E and F) together with the use of geometric margins showed to be able to mitigate some of the effects of the inter-fractional
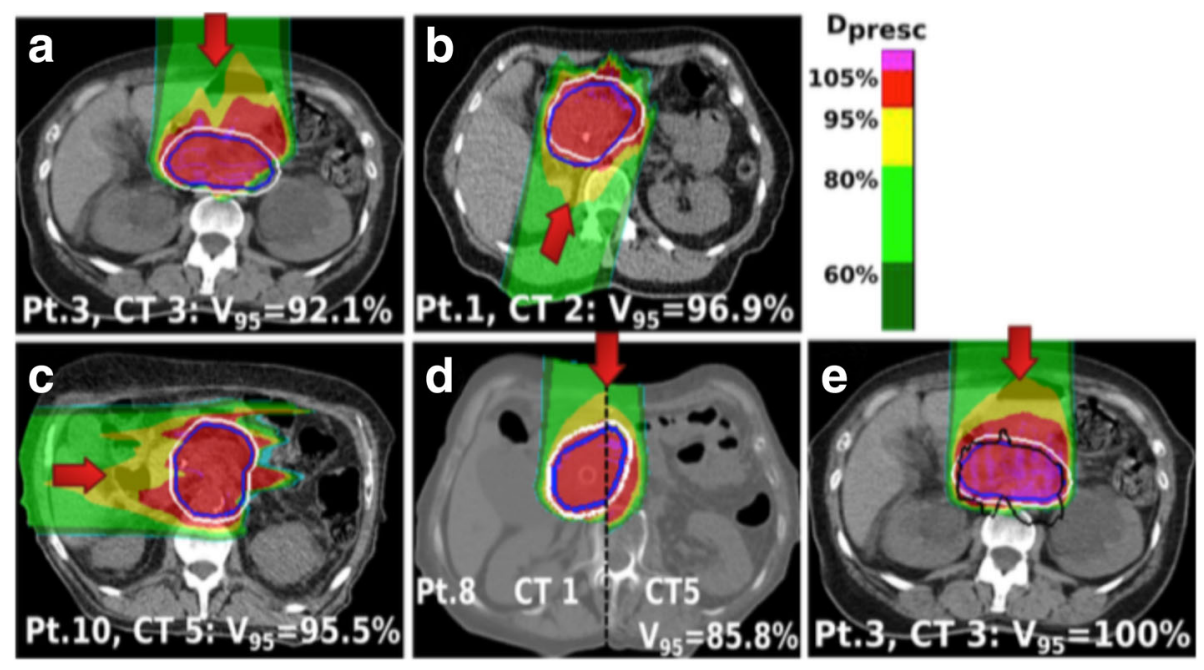

Fig. 3 Forward dose distribution of the optimized plan for the ITVGS (white contour) in one of the weekly CTs. Different inter-fractional changes are disclosed for analysis of the CTV (b/ue) V95 CTV: (a) Patient 3, for which the use of an anterior beam results in CTV under-dose due to changes in the bowel/stomach, (b) Patient 6, the oblique posterior beam does not overcome the tumor shift and deformation. c Patient 10, irradiation with a lateral right beam, where the range changes resulted from the bowel and liver position led to health tissues overdose. $\mathbf{d}$ Patient 8, an anterior beam is not robust to the accentuated weight loss (visible the comparison of the distribution in the first and last week CT). e Dose distribution for the same $\subset T$ and patient as in the figure a) but showing the retrospectively determined ITV $\mathrm{WEPL}_{\text {(black contour) in comparison to the ITV }}$ S 
Table 1 V95 $\mathrm{CTV}$ mean and standard deviation over all patients and per beam incidences and ITV-concepts

\begin{tabular}{|c|c|c|c|c|c|c|}
\hline \multicolumn{7}{|l|}{ V95 (mean \pm st.deviation) of the CTV } \\
\hline ITV Concept \& Beam Configuration(A-F) & A & $\mathrm{B}$ & C & $\mathrm{D}$ & E & $\mathrm{F}$ \\
\hline $\mathrm{ITV}_{0}$ & $95.6 \pm 2.8$ & $96.4 \pm 2.7$ & $88.7 \pm 7.0$ & $90.8 \pm 5.6$ & $98.0 \pm 2.0$ & $98.9 \pm 1.5$ \\
\hline $\mathrm{ITV}_{3}$ & $98.1 \pm 1.7$ & $98.6 \pm 1.6$ & $92.6 \pm 6.4$ & $94.0 \pm 4.2$ & $98.9 \pm 1.7$ & $99.5 \pm 1.2$ \\
\hline $\mathrm{ITV}_{\mathrm{HU}}$ & $98.1 \pm 1.6$ & $98.6 \pm 1.6$ & $92.6 \pm 6.3$ & $94.2 \pm 4.1$ & $99.1 \pm 1.4$ & $99.5 \pm 1.2$ \\
\hline $\mathrm{ITV}_{5}$ & $99.2 \pm 1.2$ & $99.4 \pm 1.2$ & $95.8 \pm 5.2$ & $96.7 \pm 3.1$ & $99.4 \pm 1.3$ & $99.6 \pm 1.2$ \\
\hline ITV & $99.2 \pm 1.2$ & $99.4 \pm 1.2$ & $95.8 \pm 5.1$ & $96.8 \pm 3.0$ & $99.4 \pm 1.2$ & $99.6 \pm 1.2$ \\
\hline ITVAsy & - & - & $97.5 \pm 3.9$ & $98.1 \pm 2.1$ & - & - \\
\hline
\end{tabular}

range changes, Table 1 and Fig. 4, where the use of the ITV $_{\mathrm{G} 5}$ was enough to keep the mean V95 $\mathrm{CTV}$ above $99 \%$. From the outlier of the Fig. $4 \mathrm{a}$ ) is concluded that even for the more robust geometries there are cases of patients and isolated fractions with dose degradation. The analysis of these particular cases, was found to correspond to patients with changes in the tumor volume and COM (e.g. Fig. 3b). To overcome these situations the use of two oblique posterior beams showed to be more robust.

Other analyzed option to increase the plan robustness was the use of symmetric range-equivalent margins ITV $_{\mathrm{HU}}$. The results (Table 1 and Fig. 4a) showed that the used of 3 and $5 \mathrm{~mm}$ HU-uniform was not sufficient to mitigate the extreme range changes in the anterior and lateral beam direction, without significant improvement over the geometric concept.

\section{Asymmetric margin analysis}

The obtained ITV $\mathrm{WEPL}_{\text {that }}$ includes the range variations along the weekly-CTs, represent how asymmetrically the ITV needs to be defined to maintain the CTV coverage. A representative example is shown in 3e.

The ITV size and shape necessary to keep the CTV coverage does not need to be volumetrically larger than a
$5 \mathrm{~mm}$ expansion with the ITV $\mathrm{IWEPL}_{\text {velume }}(-26.0 \pm 6.3) \%$ smaller than the ITV $\mathrm{G}_{\mathrm{G}}$, as result of tight margins in the lateral direction to the beam and a margin increase for the non-robust beam geometries in the beam direction (depth).

The margin size and expansion direction were assessed from the $\mathrm{COM}$ variation and $\mathrm{HD}_{95}$ value. The mean V $95_{\text {CTV }}$ over the course of the treatment when no margins are applied was used as metric of the inter-fractional changes per beam direction. Its correlation with the variation of the DSC and the $\mathrm{HD}_{95}$ between the CTV and the

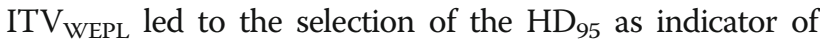
range changes due to a positive linear relation $(r=0.78)$ versus the smaller correlation coefficient of the DSC $(r=0.63)$.

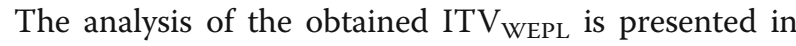
terms of median and quartiles values in the Table 2 and Fig. 5. For non-robust geometries $(\mathrm{C}$ and $\mathrm{D})$ the margin to apply to the CTV to cover the changes can be up to $10 \mathrm{~mm}$ in depth larger in the proximal edge of the beam for the most patients, while the lateral direction to the $\mathrm{BEV}$ requires less than $5 \mathrm{~mm}$. Based on these results, new plan optimizations were performed for an asymmetric ITV $\left(\right.$ ITV $\left._{\text {Asy }}\right)$ that covers $75 \%$ of the dose distributions, corresponding to a margin in the proximal direction of $10 \mathrm{~mm}$ and $7 \mathrm{~mm}$, and of $6 \mathrm{~mm}$ and $3 \mathrm{~mm}$ in the distal
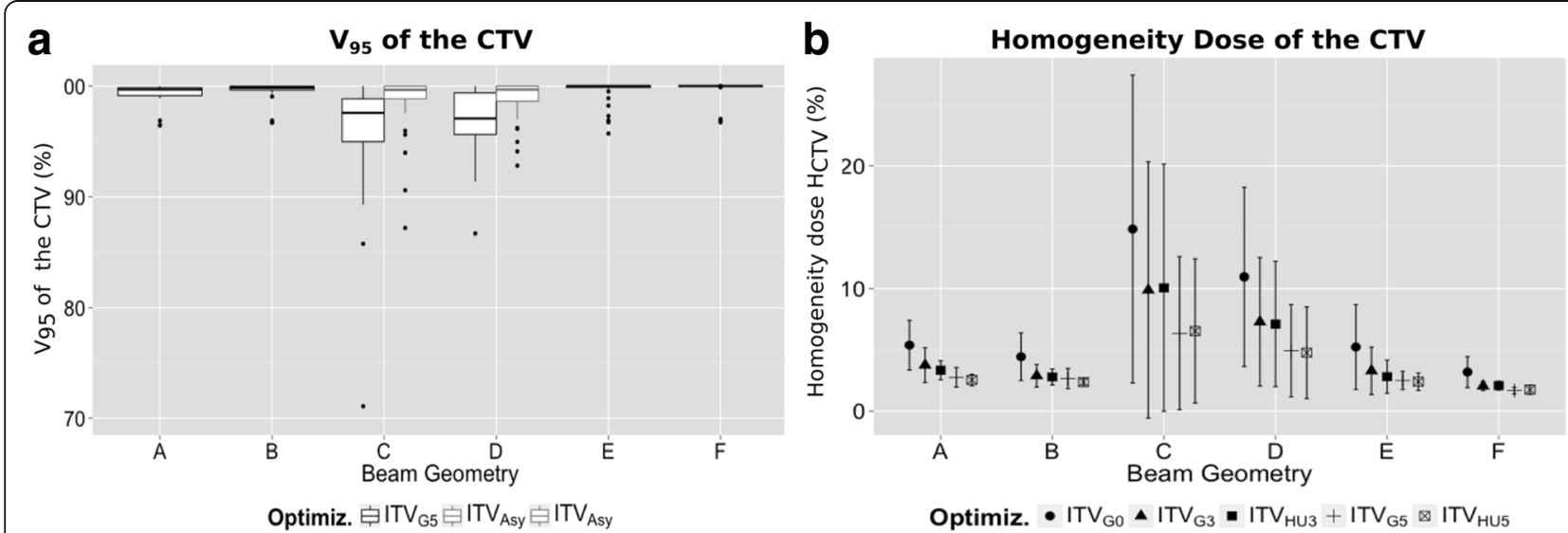

Fig. 4 Evaluation of the CTV dose from the weekly dose distribution obtained from different optimizations. a V95CTV of the plan optimized to the $I T V_{G 5}$ (dark grey boxes) and using the ITV ${ }_{\text {Asy }}$ for the non-robust geometries. Each box represents $25-75 \%$ of the data, with the median value represented as the solid line and the outlier as the dots. b Mean and standard deviation of the $\mathrm{H}_{\mathrm{CTV}}$ 


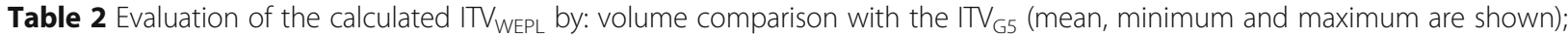
directional expansion relative to the CTV ( $\left.\mathrm{HD}_{95 \%}\right)$; $\mathrm{COM}$ variation relative to the CTV. The reported values as $\mathrm{Q}_{25 \%}$ and $\mathrm{Q}_{75 \%}$ correspond respectively to the first and third quartiles of the data

\begin{tabular}{|c|c|c|c|c|c|}
\hline \multirow{3}{*}{$\begin{array}{l}\text { Beam } \\
\text { Configuration } \\
\text { (A-F) }\end{array}$} & \multicolumn{5}{|c|}{ ITV WEPL Characterization } \\
\hline & \multicolumn{2}{|c|}{$\Delta V$ ITV $_{5}-$ ITV WEPL $_{\text {WE }}$} & \multirow{2}{*}{$\begin{array}{l}\text { CTV expansion }(\mathrm{mm}) \\
\left(\mathrm{Q}_{75 \%} \text { of } \mathrm{HD}_{95 \%}\right)\end{array}$} & \multirow{2}{*}{$\begin{array}{l}\Delta \text { COM. Depth }(\mathrm{mm}) \\
{\left[\mathrm{Q}_{25 \%} ; \mathrm{Q}_{75 \%}\right]}\end{array}$} & \multirow{2}{*}{$\begin{array}{l}\Delta \text { COM. Lateral (mm) } \\
{\left[\mathrm{Q}_{25 \%} ; \mathrm{Q}_{75 \%}\right]}\end{array}$} \\
\hline & Mean (\%) & [Min-Max] (\%) & & & \\
\hline$A, F 1$ & -28.0 & {$[-35.3,-22.6]$} & 4.5 & {$[-1.2 ;-0.3]$} & {$[-0.2 ; 0.3]$} \\
\hline$B, E 2$ & -27.9 & {$[-36.5,-22.1]$} & 4.5 & {$\left[\begin{array}{ll}-0.8 & -0.5\end{array}\right]$} & {$[0 ; 0.2]$} \\
\hline C & -16.9 & {$[-30.5,-8.4]$} & 10 & {$[-5.3 ; 2.8]$} & {$[-0.2 ; 0.5]$} \\
\hline $\mathrm{D}, \mathrm{E} 1$ & -26.9 & {$[-37.2,-15.5]$} & 6.8 & {$[-3.1 ; 0.4]$} & {$[0.1 ; 0.7]$} \\
\hline $\mathrm{F} 2$ & -28.7 & {$[-35.9,-21.4]$} & 5.0 & {$[-0.7 ; 0.7]$} & {$[-0.1 ; 0.5]$} \\
\hline
\end{tabular}

a

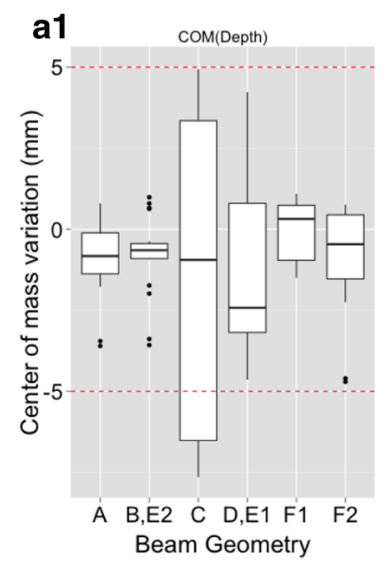

b



a2

\section{Center of mass variation}
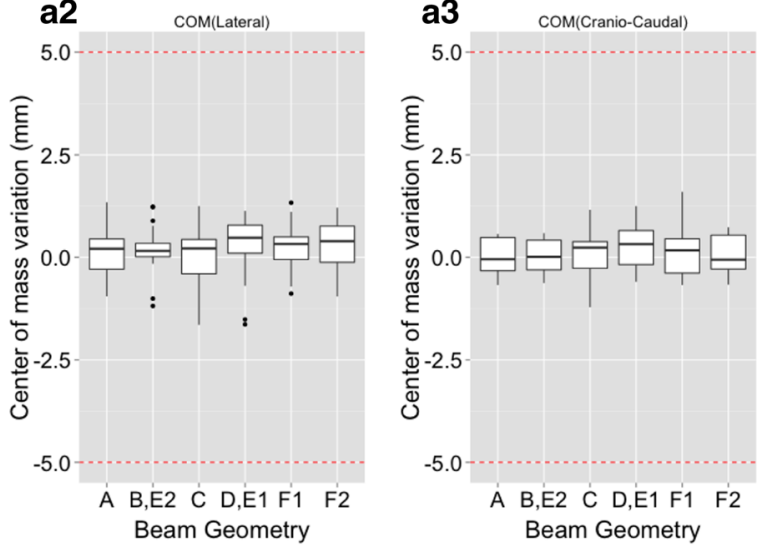

Hausdorff distance

\section{b2}

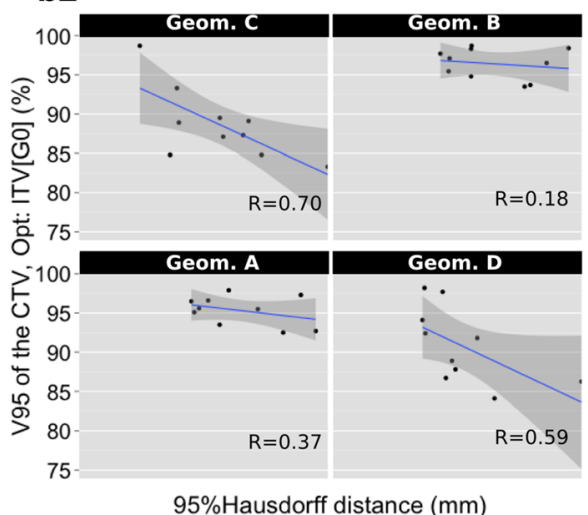

Fig. 5 (a1-a3) Variation of the ITV WEPL COM relative to the CTV in the longitudinal (a1), lateral (a2) and superior-inferior (a3) direction with respect to beam's-eye-view. b1) $\mathrm{HD}_{95 \%}$ as function of the beam geometry, with each box showing 25-75\% of the data. b2) Correlation, per beam direction, between the CTV mean dose for the weekly $C T$ s (plan optimized to the ITV $\mathrm{GO}_{\mathrm{O}}$ ) and the $\mathrm{HD}_{95}$ 
direction to cover the COM variations for the geometry $\mathrm{C}$ and $\mathrm{D}$, respectively. The delivery of these new plans to each weekly-CT, showed an improvement of the V95 $\mathrm{CTV}$, Fig. 4a.

For robust geometries the analysis showed to be suitable the use of a symmetric margin where $5 \mathrm{~mm}$ is enough for $75 \%$ of the fractions.

\section{Discussion}

For the analyzed patients the tumor inter-fractional changes are in agreement to the findings by Liu et al. [18] who report changes in the COM in the order of 1-2 mm with substantial changes in the tumor volume. Anatomical changes in the healthy tissues were also detected along the course of the treatment, with density changes in the bowel and stomach and weight loss, resulting in drastic reductions of the V95 ${ }_{\mathrm{CTV}}$ and homogeneity. Kumagai et al. [10] pointed out variations on the D95 СTV of $10 \%$ due to intra-fractional bowel changes using a passive delivery system, which matches with our results using a single beam in the anterior or lateral direction in the presence of interfractional changes. An interesting study of Houweling et al. [19] used alternatively the routinely acquired conebeam CT $(\mathrm{CBCT})$ to assess the inter-fractional changes in the patient anatomy, through dose calculations in a CT, obtained by deformable registration of the planning CT to these CBCTs. This method results in an increase of the available data but the deformable registration adds new uncertainties to the evaluation.

The influence of the range changes on dose to the OARs was not part of our analysis and further investigation need to be included especially for geometries where the OARs are in the beam distal edge.

We have demonstrated that the impact of interfractional changes can be reduced to a large extent with an adequate selection of the beam geometry and ITV-margins. For the analyzed treatment fractions and patients the use of a symmetric margin of $5 \mathrm{~mm}$ and two posterior oblique beams was enough to keep the CTV coverage above $95 \%$, with $10 \%$ of the cases with a V95 $5_{\text {CTV }}$ of $95-98 \%$. Nevertheless, cases with substantial changes in the CTV shape or location were detected, leading to a decrease in dose coverage (outliers in Fig. 4a). In these cases, treatment plan adaption or re-planning might be an option. Moreover, our results only considered uncertainties resulting from anatomy changes and the uncertainties from the patient positioning were neglected. Regarding the patient positioning, studies from Jayachandran et al. [20] showed that bone-matching registration might not accurately predict the tumor location, and therefore suggests the use of fiducial markers combined with daily soft-tissue imaging.

The definition of PTV margins is beyond the scope of this work which aimed to isolate the inter-fractional motion impact. With exception of errors from the rigid registration, other systematic errors, as example uncertainties in Hounsfield units or patient positioning, do not have an impact on our simulation results. However, they need to be taken into account for clinical implementation.

An important scope of the study was to suggest clinically feasible approaches to increase the plan robustness to account for range uncertainties. From the first attempt with a symmetric range-uniform concept, the results showed that a small margin of 3 or $5 \mathrm{~mm}$ was not enough to overcome the uncertainties from non-robust beam configurations. Hence, the use of asymmetric field specific margins was evaluated, which was already investigated by Park et al. [21] as an approach to mitigate range uncertainties. Also, Miki et al. [22] investigated the use of field-specific target and OARS but for the case of intrafractional changes. In our study, the obtained ITV $\mathrm{WWEP}_{\text {, }}$ based on Graeff et al. [11] study, preserves the CTV coverage over the set of weekly-CTs, including the interfractional anatomical changes. The obtained ITV $V_{\text {WEPL }}$ matched with the literature that suggests small lateral margins and larger margins in the direction of the low density tissues, that here correspond usually to the proximal direction [21, 23].

Note that the ITV $V_{\text {WEPL }}$ concept cannot be directly used in the clinical routine since the complete information of the inter-fractional changes along the treatment course is not readily available at the planning stage. However, we have used this approach to guide the definition of an asymmetric standard margin for future patients. The suggested asymmetric margins were tested for the non-robust geometries and showed a V95 ${ }_{\mathrm{CTV}}$ improvement face to the symmetric concept. The validation of this concept in a different cohort of patient remains to be shown and will be investigated in a future study. Furthermore, this concept could be applied clinically e.g. in a boost treatment, where several planning CTs have been obtained in the first treatment sessions with photons.

Our study was tailored to improve the treatment plan robustness against inter-fractional changes using IMPT optimized plans. However, a comparison of the plan quality using SFUD and IMPT is suggested prior to the implementation of this technique in others facilities, since the degree of plan modulation can compromise the robustness. In our study, all the plans represented reduced modulation. Therefore the results might be extended to SFUD optimizations.

Intra-fractional changes were not investigated and will require an additional expansion of the target volume [24]. Larger margins alone are not a solution for scanned ion therapy since interplay effects will have an impact on the dose coverage and homogeneity throughout the complete volume [25, 26]. Shiomi et at. [27] evaluated, how the use of specific beam directions might increase 
the plan robustness for the case of passive and active delivery systems. Moreover, a complementary approach is the use of abdominal compression to reduce the motion amplitude and additional beam rescanning to mitigate the interplay effect [28]. There is no consensus on the quantification of intra-fractional motion in pancreas $[18,29]$ among the studies, and a separated study will be conducted to assess the need of mitigation techniques. This might include the use of beam-gating [30] or other modified beam delivery strategies, as well the use of $4 \mathrm{D}$ optimized plans [31] or quantification of range variations based on daily CT imaging [32].

\section{Conclusion}

In conclusion, the combination of two oblique posterior beams for scanned carbon-ion treatment of pancreatic cancer with the simple concept of an isotropic CTV-ITV margin of $5 \mathrm{~mm}$ can substantially reduce the dosimetric impact of inter-fractional changes and yields to acceptable dose coverage for most patients and fractions. The use of WEPL-based margins can reduce the required margins even more, but this technique will require a daily monitoring of the anatomical changes. Therefore, range variations may be large and routine soft-tissue imaging and adaption strategies will likely improve the treatment. Populationbased asymmetric ITV margins may be a feasible clinical strategy to account for density changes and to reduce the irradiated volume as well as to assess the validity of the selected margins for a defined beam path direction.

\section{Additional file}

Additional file 1: Supplementary Figure and Tables. (DOC 831 kb)

\begin{abstract}
Abbreviations
COM: Center of mass; CT: Computed tomography; CTV: Clinical target volume; DSC: Dice similarity coefficient; GTV: Gross tumor volume;

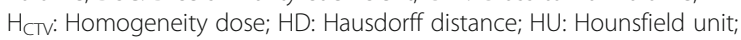
IMPT: Intensity-modulated particle therapy; ITV: Internal target volume; PTV: Planning target volume; r: Person correlation index; RBE: Relative biological effectiveness; TPS: Treatment planning system; WEPL: Water-equivalent range path
\end{abstract}

\section{Acknowledgments}

Not applicable.

\section{Funding}

The study was financed by the German Research Foundation, clinical research group 214

\section{Availability of data and material}

The datasets used and analysed during the current study are available from the corresponding author on reasonable request.

\section{Authors' contributions}

VB, OJ and SEC participated in the design and plan of the study. SEC contoured the target volumes and contributed to the data collection. VB and DR were responsible for the treatment plans optimization and physical evaluation. VB performed the statistical analysis. VB and DR drafted the manuscript and all authors revised it critically. All authors read and approved the final manuscript.

\section{Competing interest}

The authors declare that they have no competing interests.

\section{Consent for publication}

Not applicable.

Ethics approval and consent to participate

Not applicable.

\section{Publisher's Note}

Springer Nature remains neutral with regard to jurisdictional claims in published maps and institutional affiliations.

\section{Author details}

${ }^{1}$ Hospital for Radiooncology and Radiation Therapy, Heidelberg University Hospital, Heidelberg, Germany. ${ }^{2}$ University Clinic of Erlangen, Erlangen, Germany. ${ }^{3}$ Heidelberg lon-Beam Therapy Center, Heidelberg, Germany. ${ }^{4}$ Klinikum rechts der Isa, Technische Universität München, Muniche, Germany. ${ }^{5}$ GSI Helmholtz Centre for Heavy lon Research, Darmstadt, Germany.

${ }^{6}$ Division Medical Physics in Radiation Oncology, German Cancer Research Center, Heidelberg, Germany.

Received: 30 November 2016 Accepted: 30 May 2017

Published online: 08 June 2017

\section{References}

1. Li D, Xie K, Wolff R, Abbruzzese JL. Pancreatic cancer. Lancet. 2004;363:1049-57.

2. Kessel KA, Habermehl D, Jäger A, Floca RO, Zhang $L$, Bendl $R$, et al. Development and validation of automatic tools for interactive recurrence analysis in radiation therapy: optimization of treatment algorithms for locally advanced pancreatic cancer. Radiat Oncol. 2013;8:138.

3. Durante M, Loeffler JS. Charged particles in radiation oncology. Nature reviews. Clinical oncology 7.1 (2010): 37.

4. Tsujii H, Kamada T, Shirai T, Noda K, Tsuji H, Karasawa K. Carbon-lon Radiotherapy: Principles, Practices, and Treatment Planning. Springer Science \& Business Media. 2013.

5. Combs SE, et al. Phase I study evaluating the treatment of patients with locally advanced pancreatic cancer with carbon ion radiotherapy: the PHOENIX-01 trial. BMC cancer. 13.1 (2013): 419.

6. Matsui $Y$, Asano T, Kenmochi T, Iwakawa M, Imai T, Ochiai T. Effects of carbon-ion beams on human pancreatic cancer cell lines that differ in genetic status. Am J Clin Oncol. 2004:27:24-8.

7. Yamada S, Shinoto M, Imada H, Yasuda S, Kamada T, Tsujii H. Carbon ion radiotherapy for patients with gastrointestinal cancer. PTCOG 49. Chiba, Japan: Ref Type: Generic; 2010;

8. Dalal S, et al. "Relationships among body mass index, longitudinal body composition alterations, and survival in patients with locally advanced pancreatic cancer receiving chemoradiation: a pilot study." J Pain Symptom Manage. 2012;44.2:181-91.

9. Mori S, Hara R, Yanagi T, Sharp GC, Kumagai M, Asakura H, et al. Four-dimensional measurement of intrafractional respiratory motion of pancreatic tumors using a 256 multi-slice CT scanner. Radiother Oncol. 2009:92:231-7.

10. Kumagai M, Hara R, Mori S, Yanagi T, Asakura H, Kishimoto R, et al. Impact of intrafractional bowel gas movement on carbon ion beam dose distribution in pancreatic radiotherapy. Int J Radiat Oncol Biol Phys. 2009;73:1276-81.

11. Graeff C, Durante M, Christoph B. Motion mitigation in intensity modulated particle therapy by internal target volumes covering range changes. Med Phys. 2012;39.10:6004-13.

12. Krämer M, Scholz M. Treatment planning for heavy-ion radiotherapy: calculation and optimization of biologically effective dose. Phys Med Biol. 2000:45:3319-30.

13. Krämer M, Jäkel $O$, Haberer $T$, Kraft G, Schardt D, Weber U. Treatment planning for heavy-ion radiotherapy: physical beam model and dose optimization. Phys Med Biol. 2000;45:3299-317.

14. Scholz M, Kraft G. Track structure and the calculation of biological effects of heavy charged particles. Adv Space Res. 1996;18:5-14 [cited 2014 Aug 20]. 
15. Emami B, Lyman J, Brown A, Coia L, Goitein M, Munzenrider JE. Tolerance of normal tissue to therapeutic irradiation. Int J Radiat Oncol Biol Phys. 1991;21:109-22.

16. Birkfellner W, et al. Proceedings of the first MICCAI workshop on ImageGuidance and Multimodal Dose Planning in Radiation Therapy. (2012):137.

17. Elhawary H, Oguro S, Tuncali K, Morrison P, Tatlu Se, Shyn P, et al. Multimodality Non-Rigid Image Registration for Planning, Targeting and Monitoring during CT-guided Percutaneous Liver Tumor Cryoablation. 2011;17:1334-44.

18. Liu F, et al. Characterization and management of interfractional anatomic changes for pancreatic cancer radiotherapy. International Journal of Radiation Oncology* Biology* Physics. 2012;83.3 :e423-e429.

19. Houweling AC, Fukata K, Kubota Y, Shimada H, Rasch CRN, Ohno T, et al. The impact of interfractional anatomical changes on the accumulated dose in carbon ion therapy of pancreatic cancer patients. Radiother Oncol. 2016;1 19:319-25.

20. Jayachandran P, Minn AY, Van Dam J, Norton JA, Koong AC, Chang DT. Interfractional uncertainty in the treatment of pancreatic cancer with radiation. Int J Radiat Oncol Biol Phys. 2010;76:603-7.

21. Park PC, Zhu XR, Lee AK, Sahoo N, Melancon AD, Zhang L, et al. A beam-specific planning target volume (PTV) design for proton therapy to account for setup and range uncertainties. Int J Radiat Oncol Biol Phys. 2012;82:e329-36.

22. Miki K, Mori S, Shiomi M, Yamada S. Gated carbon-ion scanning treatment for pancreatic tumour with field specific target volume and organs at risk. Phys Med. 2016;32:1521-8.

23. Engelsman M, Kooy HM. Target volume dose considerations in proton beam treatment planning for lung tumors. Med Phys. 2005;32:3549-57.

24. Solla I, Zucca S, Possanzini M, Piras S, Pusceddu C, Porru S, et al. Free breathing conformal irradiation of pancreatic cancer. J. Appl. Clin. Med. Phys., Vol. 14, No. 4, 2013. 2014;14:1-8.

25. Bert C, Grözinger SO, Rietzel E. Quantification of interplay effects of scanned particle beams and moving targets. Phys Med Biol. 2008;53:2253-65.

26. Bert C, Rietzel E. 4D treatment planning for scanned ion beams. Radiat Oncol. 2007:2:24.

27. Shiomi M, Mori S, Shinoto M, Nakayama Y, Kamada T, Yamada S. Comparison of carbon-ion passive and scanning irradiation for pancreatic cancer. Radiother Oncol. 2016:119:326-30.

28. Bert C, Durante M. Motion in radiotherapy: particle therapy. Phys Med Biol. 2011:56:R113-44.

29. Wysocka B, Kassam Z, Lockwood G, Brierley J, Dawson LA, Buckley CA, et al. Interfraction and respiratory organ motion during conformal radiotherapy in gastric cancer. Int J Radiat Oncol Biol Phys. 2010;77:53-9.

30. Mori S, Yanagi T, Hara R, Sharp GC, Asakura H, Kumagai M, et al. Comparison of respiratory-gated and respiratory-ungated planning in scattered carbon ion beam treatment of the pancreas using four-dimensional computed tomography. Int J Radiat Oncol Biol Phys. 2010;76:303-12.

31. Graeff C. Motion mitigation in scanned ion beam therapy through 4D-optimization. Phys Med. 2014:30:570-7.

32. Mori S, Chen GTY. Quantification and Visualization of Charged Particle Range Variations. Int J Radiat Oncol Biol Phys. 2008;72:268-77.

\section{Submit your next manuscript to BioMed Central and we will help you at every step:}

- We accept pre-submission inquiries

- Our selector tool helps you to find the most relevant journal

- We provide round the clock customer support

- Convenient online submission

- Thorough peer review

- Inclusion in PubMed and all major indexing services

- Maximum visibility for your research

Submit your manuscript at www.biomedcentral.com/submit

) Biomed Central 